\title{
Tan antiguos como su misma creación, mitos de las vacunas y la vacunación
}

\author{
As old as its creation, myths about vaccines and vaccination

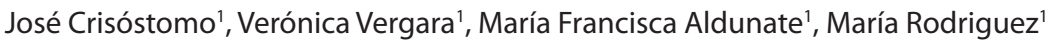

Estudios científicos coinciden en que la vacunación ha sido uno de los diez principales logros de la salud pública en el siglo XX (CDC Estados Unidos, 1999). Después del agua potable, las vacunas se consideran como la mejor defensa contra las enfermedades infecciosas y como la medida de salud pública más eficaz en reducir la mortalidad y las secuelas provocadas por las enfermedades inmunoprevenibles, así como un importante aporte a la equidad en el acceso a la salud (Saldaña et al., 2015; Valenzuela, 2020). Sin embargo, la oposición a las vacunas ha existido desde prácticamente los inicios de la vacunación. Estas críticas han sido diversas, abordando tanto el ámbito sanitario, científico y político. En Estados Unidos e Inglaterra, a mediados y fines del siglo XIX, con la aplicación de la vacuna contra la viruela, se formaron las llamadas ligas antivacunas, como la Sociedad estadounidense antivacunas, que fue fundada en 1879 , seguida de la Liga contra la vacunación obligatoria de Nueva Inglaterra (1882) y muchas más a lo largo de los años. En épocas recientes, estas dudas también han estado presentes, como las controversias generadas antes de la pandemia, que hicieron dudar sobre la seguridad, eficacia y calidad de las vacunas contra la difteria, tétanos y pertussis. Otro aspecto controvertido ha sido el empleo del mercurio utilizado en preservantes como el timerosal el cual, fue asociado al autismo, hecho desmentido en numerosas publicaciones y estudios científicos (Hurley et al., 2010).

Las vacunas contra COVID-19, como todas las vacunas y medicamentos utilizados en nuestro país, pasan por un riguroso proceso de evaluación de las diversas etapas de su desarrollo, antes de su utilización en la población. Estas etapas incluyen extensos estudios preclínicos y clínicos que incorporan desde modelos celulares in vitro, animales de distintas especies, hasta miles de personas, de diferente rango etario, etnias, género y condiciones de salud. Estos ensayos son específicamente diseñados para establecer su eficacia e identificar problemas de seguridad de estos productos farmacéuticos (Instituto de Salud Pública de Chile, 2020). Como todo medicamento o intervención sanitaria, las vacunas no están exentas de presentar eventos adversos tras su administración. Algunos de ellos pueden llegar incluso a ser serios, pero afortunadamente su frecuencia es muy rara; aun así, el beneficio que aportan las vacunas al evitar la aparición de enfermedades potencialmente mortales, es mucho mayor (Saldaña et al., 2015; Centers for Disease Control and Prevention, 2020). De esta manera, el objetivo de estos estudios, es demostrar que, al utilizar este tipo de vacunas, el beneficio es superior al riesgo. Toda esta información de seguridad, calidad y eficacia ha sido corroborada, analizada y discutida por expertos externos e internos del Instituto de Salud Pública, para todas las vacunas autorizadas y administradas para su uso en emergencia en nuestro país. Lo anterior ha sido corroborado por otras agencias internacionales, como la Food and Drug Administration (FDA) en Estados Unidos, la European Medicines Agency (EMA) de la Unión Europea, y la Organización Mundial de la Salud (OMS).

Respecto a nuevas tecnologías en desarrollo y producción de vacunas contra COVID-19, como la basada en ácido ribonucleico mensajero (ARNm) y vectores virales, comentar que la primera tecnología ha sido estudiada por más de una década, incluyendo el desarrollo de vacunas contra el virus del Zika, rabia e influenza (Pardi et al., 2018). Por otra parte, la estrategia de utilizar vectores virales como vehículos para medicamentos, es aún más antigua y ha sido utilizada para el desarrollo de vacunas y otros tipos medicamentos como la terapia génica (Robbins \& Ghivizzani, 1998). Las vacunas desarrolladas con estas tecnologías han sido estudiadas en profundidad, demostrando producir inmunidad por un largo período de tiempo, siendo los efectos secundarios comunes $y$ similares a todas las vacunas y otros medicamentos, que en su gran mayoría no son graves. Ninguna de estas vacunas contiene virus

(1) Instituto de Salud Pública de Chile, Departamento Agencia Nacional de Medicamentos. Autor de correspondencia: jcrisostomo@ispch.cl 
vivos y no interfieren con el ADN humano, como se ha planteado en una serie de artículos y noticias falsas, las cuales se encuentran totalmente desmentidas por publicaciones científicas recientes (Smits et al., 2021).

Finalmente, debe considerarse que el perfil de riesgos de una vacuna y de cualquier medicamento, es dinámico (Saldaña et al., 2015) pudiendo así variar en el tiempo. Esto principalmente por las limitaciones que existen en los estudios clínicos, en donde, por diversos factores, no es posible detectar los eventos adversos de baja frecuencia (Mead \& Tagliavini, 2018). Por ello, una vez que las vacunas contra COVID-19 cuentan con la autorización de uso de emergencia en nuestro país, el Instituto de Salud Pública (ISP), a través de su Centro Nacional de Farmacovigilancia, realiza un monitoreo constante de su seguridad, con el objetivo de garantizar que los beneficios de su administración son mayores que los posibles riesgos. De lo contrario, el ISP tiene facultades para restringir o suspender su uso.

En conclusión, las vacunas contra la COVID-19 son una herramienta importante para controlar la pandemia, ya que éstas protegen contra la enfermedad al inducir inmunidad contra el virus SARSCoV-2 que la causa. Se ha demostrado que, reducen el riesgo de contraer formas graves de COVID-19, evitando consecuencias graves para la salud (Organización Mundial de la Salud, 2020). EI ISP garantiza el cumplimiento de los estándares de calidad, seguridad y eficacia, aun después de su autorización, dando así tranquilidad a la población, que los beneficios superan en gran medida, los potenciales riesgos asociados a la vacunación.

\section{Referencias}

CDC Estados Unidos. (1999). Ten Great Public Health Achievements -- United States, 1900-1999. CDC. Accedido en https://www.cdc.gov/ mmwr/preview/mmwrhtml/00056796.htm el 17 de agosto de 2021 .

Centers for Disease Control and Prevention. (2020). Overview, History, and How the Safety Process Works. CDC. Accedido en https:// www.cdc.gov/vaccinesafety/ensuringsafety/history/index.html el 19 de agosto de 2021.
Hurley AM, Tadrous M. \& Miller ES. (2010) Thimerosal-containing vaccines and autism: a review of recent epidemiologic studies. The journal of pediatric pharmacology and therapeutics: JPPT: the official journal of PPAG 15: 173-81.

Instituto de Salud Pública de Chile. (2020). Fases de desarrollo de las vacunas. Accedido en https://www.ispch.cl/anamed/farmacovigilancia/vacunas/fases-de-desarrollo-de-las-vacunas/ el 19 de agosto de 2021.

Mead S. \& Tagliavini F. (2018). Chapter 24 - Clinical trials (Vol. 153). Maurizio Pocchiari, Jean Manson. 10.1016/B978-0-444-639455.00024-6

Organización Mundial de la Salud. (2020). Enfermedad por el coronavirus (COVID-19): Vacunas. WHO. Accedido en https:// www.who.int/es/news-room/q-a-detail/coronavirus-disease-(covid-19)-vaccines?adgroupsurvey $=\% 7$ badgroupsurvey $\% 7 \mathrm{~d} \& g c l i-$ d=CjwKCAjwmeilBhA6EiwA-uaeFZyackMYO6U_zfXGsOr9pWeDXRbGal97o-UATs-kPeRV-nL3sG4c8BoCqbAQAvD_BwE el 18 de Agosto de 2021.

Pardi N, Hogan MJ, Porter FW. \& Weissman D. (2018). mRNA vaccines - a new era in vaccinology. Nature Reviews Drug Discovery $17,261-279$

Robbins PD. \& Ghivizzani SC. (1998). Viral vectors for gene therapy. Pharmacol Ther. 80, 35-47.

Saldaña A, Avendaño M, Silva X. \& Moya A. (2015). Seguridad de las Vacunas. Boletín de farmacovigilancia vacunas, 1 . Accedido en https://www.ispch.cl/newsfarmacovacunas/01/images/04seguridadvacunas.pdf el 18 de Agosto de 2021.

Smits N, Rasmussen J. \& Bodea GO. (2021). No evidence of human genome integration of SARS-CoV-2 found by long-read DNA sequencing. Cell Reports 36, 109530

Valenzuela MT. (2020). Importancia de las vacunas en salud pública: hitos y nuevos desafíos. Revista Médica Clínica las Condes 31, 233-239. 